\title{
Costing hospital resources for stroke patients using phase-type models
}

\author{
Jennifer Gillespie • Sally McClean • Bryan Scotney • \\ Lalit Garg • Maria Barton • Ken Fullerton
}

Received: 25 August 2010 / Accepted: 6 June 2011

(C) Springer Science+Business Media, LLC 2011

\begin{abstract}
Optimising resources in healthcare facilities is essential for departments to cope with the growing population's requirements. An aspect of such performance modelling involves investigating length of stay, which is a key performance indicator. Stroke disease costs the United Kingdom economy seven billion pounds a year and stroke patients are known to occupy long periods of time in acute and long term beds in hospital as well as requiring support from social services. This may be viewed as an inefficient use of resources. Thrombolysis is a therapy which uses a clot-dispersing drug which is known to decrease the institutionalisation of eligible stroke patients if administered $3 \mathrm{~h}$ after incident but it is costly to administer to patients. In this paper we model the cost of treating stroke patients within a healthcare facility using a mixture of Coxian phase type model with multiple absorbing states. We also discuss the potential benefits of increasing the usage of thrombolysis and if these benefits balance the expense of administering the drug.
\end{abstract}

J. Gillespie $(\bowtie) \cdot S$. McClean · B. Scotney $\cdot$ L. Garg $\cdot$ M. Barton

School of Computing and Information Engineering,

University of Ulster,

Coleraine Campus, South Building,

Coleraine, Northern Ireland, UK

e-mail: gillespie-j@email.ulster.ac.uk

S. McClean

e-mail: si.mcclean@ulster.ac.uk

B. Scotney

e-mail: bw.scotney@ulster.ac.uk

K. Fullerton

School of Medicine, Dentistry and Biomedical Sciences,

Queen's University,

Belfast, UK

e-mail: ken.fullerton@belfasttrust.hscni.net
Keywords Patient flow Phase-type models $\cdot$ Healthcare modelling $\cdot$ Thrombolysis $\cdot$ Stroke disease

\section{Introduction}

With more budget cuts expected within the next few years, performance management within healthcare facilities is more important than ever as there will be an increasing demand on the resources available in the departments [42]. By modelling and analyzing healthcare facilities a more efficient and cost-effective service may be achieved. The United Kingdom Department of Health (UK DoH) introduced the NHS Institute for Innovation and Improvement in 2005 to support a transformation of the United Kingdom National Health Service (UK NHS) [9, 10]. Their aim was to provide a world class healthcare service for the UK through innovation, improvement and adoption of best practice. Due to the number of different pathways patients can take from admission to discharge, healthcare facilities can be seen as part of a complex system [18]. Therefore, to achieve a better healthcare service, comprehensive performance analysis allied to key performance indicators can help greatly with regard to improvement and transformation of the service. Key performance indicators measure the progress towards operational goals that have been set in place, and include measuring length of stay (LoS), mortality rates, readmission rates and day case rates [9].

Stroke disease is placing increased strain on healthcare facilities due to its debilitating nature and complexity. In the UK the disease is costing the economy $£ 7$ billion a year [36] which is approximately $7 \%$ of the UK NHS budget [8]. This is mainly incurred by prolonged periods of time spent in acute and long-term hospital beds and community care [39]. This may be seen as an inefficient use of resources 
and much research has been carried out to decrease patients' length of stay and reduce health and social care costs [37-39].

Thrombolysis is a therapy which uses a clot dispersing drug for appropriate stroke patients. It can decrease the short term hospitalisation of eligible stroke patients if administered within $3 \mathrm{~h}$ after incident [38] and also has the potential to increase the proportion of patients who are discharged to their usual residence instead of going to private nursing homes. To be eligible for thrombolytic therapy in the UK a patient must have a diagnosis of ischemic stroke and be 80 years old or less. Thrombolytic therapy with intravenous altephase (rt-pa) has been licensed for use since 2003 for treatment of ischemic stroke and is currently administered to $9.5 \%$ of eligible patients in the UK [36]. The small percentage of patients receiving the treatment is partly due to thrombolytic therapy being expensive to administer and it puts increased strain on the resources of the department.

In this paper we will model the LoS of stroke patients using a mixture of Coxian phase-type models with multiple absorbing states. Our approach extends the methodology presented in McClean et al. (2011), [25], to include costs and applies it to stroke patients who are eligible to receive thrombolysis. By changing the proportion of eligible patients who receive thrombolysis we can determine whether the benefits of thrombolysis outweigh the expense of implementing an expensive drug. In addition, we have developed a methodology to find the maximum cost that the drug could be increased to before the benefits no longer balance the cost of administration.

The paper is outlined as follows; in Section 2 a brief description of some of the methods used in the past to model LoS will be described, including Markov models which are the basis for defining phase-type models used for the analytic model in this paper. In addition, we will demonstrate how these methods have been used to model some systems within healthcare. For a more detailed account of how LoS has been modelled in the past and the directions for the future see Marshall, et al. [30]. Section 3 will describe the methodology presented by McClean et al. (2011), [25], before extending the model to include costs. The model will be applied to eligible stroke patients in Section 4 before presenting the results in Section 5. We will conclude the paper in Section 6 and discuss any further work.

\section{Modelling length of stay}

Modelling LoS in hospitals is a key aspect of characterising patient flow and has been used by many researchers to find solutions for problematic healthcare departments and processes $[5,12-15,22-32,40]$. In the past, methods such as mixed-exponential distributions, Markov models, compartmental models and phase-type distributions have been used to model LoS in healthcare facilities. In this section we will describe some ways in which LoS has been modelled in the past and also provide evidence of where the models have been applied within a healthcare environment.

\subsection{Markov models}

Markov models are characterised as a discrete or continuous time process in which an individual, here a patient, experiences a set of changes in a sequence of times or states [20]. It is a commonly used approach for stochastic modelling and is the basis for defining phase-type models (see Section 2.4). To define a Markov model three descriptors are required. The first is the state space, $S$, which is the set of states and can include both transient and absorbing states. A transient state is such that once reached the probability of ever returning is less than one. An absorbing state is one which, once reached, is never left. The second descriptor is the initial state probability distribution p. This de-termines which state will be the initial state. The third descriptor is the infinitesimal generator $\mathbf{Q}$. which provides the transition rates between states. In the infinitesimal generator the rows represent the state we move from and the columns represent the state we move to [11, 21, 34]. The diagonal entries of an infinitesimal generator are negative and represent outflows from that state. The in-finitesimal generator is also known as the transition matrix. To see an example of a finite-state continuous-time Markov chain where each of the descriptors are provided see Example 1 as follows.

Example 1: Figure 1 provides an example of a Finite-State Continuous-Time Markov Model with 3 transient states (1, 2 and 3$)$ and 1 absorbing state (0).

Let $S$ represent the state space. Therefore, the state space is $S=\{0,1,2,3\}$ where states 1 , 2 and 3 are transient states and state 0 is an absorbing state.

The initial state probability distribution $\mathbf{p}$ is:

$\mathbf{p}=\left(\frac{1}{10}, \frac{1}{5}, \frac{2}{5}, \frac{3}{10}\right)$

The infinitesimal generator $\mathbf{Q}$ is:

$$
\mathbf{Q}=\left(\begin{array}{cccc}
0 & 0 & 0 & 0 \\
3 & -12 & 2 & 7 \\
0 & 6 & -11 & 5 \\
5 & 0 & 8 & -13
\end{array}\right)
$$


Some examples of when Markov models have been used to analyse healthcare systems are as follows. Taylor, et al. (1998) developed a continuous-time Markov model which was used to find the expected number of geriatric patients for a cohort and which state they resided in at any particular time [40]. Christodoulou and Taylor (2001) used a hidden Markov process with discrete states to find if the covariates of geriatric patients had an effect on the parameter estimates for an exponential fit [5]. A continuous time Markov model has also been used to model the LoS of patients in residential and nursing home care [32]. By including costs in a discrete Markov model an estimate of the total cost to treat geriatric patients was obtained by McClean, et al. [22]. Also see [6, 16, 22-25, 40, 43] for further examples.

\subsection{Exponential and mixed-exponential distributions}

The exponential distribution is the most commonly used distribution in stochastic modelling, probably for the reasons that it requires estimation of a single parameter and is mathematically tractable. It is also the only distribution which has the memoryless property. Consider a non-negative random variable $\boldsymbol{T}$ which is distributed according to an exponential distribution with parameter $\lambda>0$. The probability density function of $\boldsymbol{T}$ is defined as:

$f(t)=\lambda e^{-\lambda t}$

where $t \geq 0$ [20]. The memoryless property means that if a patient's lifetime can be represented by an exponential distribution then the chance of an event, like discharge from hospital, occurring at time $t$ is not affected by the events that have occurred in the patient's past, for example length of stay in hospital. Using the exponential distribution to model key performance indicators such as LoS allows for simple expressions. However, the disadvantage of the distribution is its lack of versatility due to its single parameter which can lead to a poor representation of the distribution when fitted to a dataset [11]. Some healthcare processes are better modelled using a mixed exponential distribution. The probability density function for the length of stay distribution is then given by:

$f(t)=\sum_{i=1}^{k} \rho_{i} e^{-\lambda_{i} t}$

where $t \geq 0, \rho_{i}, \lambda_{i} \geq 0$ for $i=1, \ldots k$ and $\sum_{i=1}^{k} \rho_{i}=1$.

However, in many situations a two term mixed exponential distribution is sufficient to model the length of stay distribution and can adequately represent the heterogeneity [19].

Millard in 1989 [32] observed that occupancy time of certain types of patients are better modelled using mixed exponential distributions. He found that geriatric patients flowing from acute to long term care are better represented by a two term mixed exponential distribution than by a single exponential distribution. Harrison [19] also uses mixed exponential distributions to model the flow of patients through a small private hospital in America. Mixed exponential distributions can be seen as a special type of phase-type model which will be explained in Section 2.4.

\subsection{Compartmental models}

Compartmental modelling is used to break down a complex system into separate stages so that the relationship between the stages can be analysed [24]. Such compartmental models may be deterministic or stochastic; in the latter case they can be described using a continuous time Markov model. Therefore, compartmental modelling is often used for modelling healthcare systems as it breaks down the Markov states into compartments which can be analysed separately, for example, modelling acute care and long term care in hospital can be seen as a two compartment model.

McClean and Millard (1998) describe how a three compartment model can be used to model acute, rehabilitative and long stay care of geriatric patients [24]. Caulkins et al. (2007) showed that a five state compartmental model

Fig. 1 A finite-state continuous time Markov model

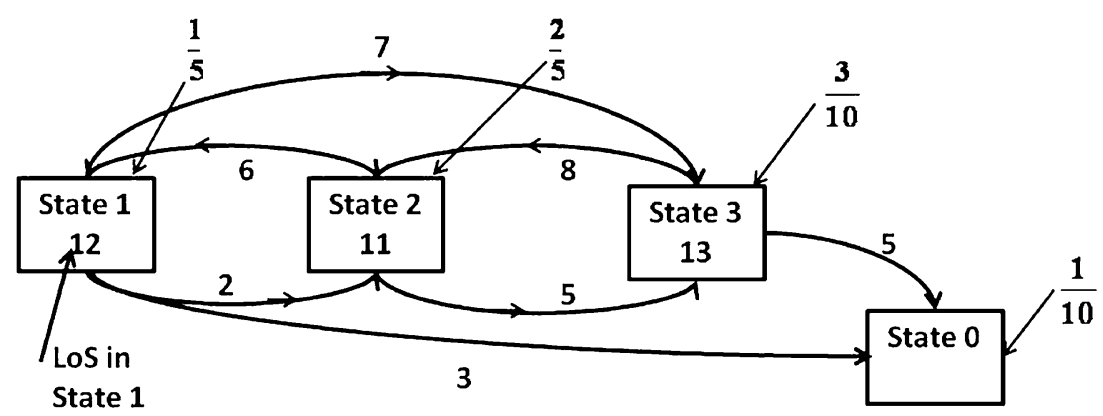


could reasonably reproduce the trends of Australian illicit drug use [4].

\subsection{Phase-type models}

Phase-type models were first introduced by Neuts in 1975 [33] and can be defined using the finite state continuous time Markov model. Consider a finite-state continuous time Markov model (see Section 2.1) with a state space $S=$ $\{0,1,2, \ldots, k\}$ where state 0 is an absorbing state. Let the initial state probability distribution be $\mathbf{p}=\left(\mathrm{p}_{0}, \mathrm{p}_{1}, \ldots, \mathrm{p}_{k}\right)$ where $\sum_{i=1}^{k} p_{i}=1$ and the infinitesimal generator is $\mathbf{Q}$. The general phase-type distribution is defined as the time to absorption of the finite-state continuous-time chain, where there is a single absorbing state and the process starts in a transient state [11, 33].

Phase-type distributions are a very versatile class of distributions and can be used to fit a distribution to statistical data by matching moments or alternatively using Maximum Likelihood estimation. They are also generalisations of a number of well-known survival distributions such as the exponential, special Erlang and general Erlang, as well as providing a good approximation for many other distributions [12, 27, 31]. In addition phase-type models can replace numerical integration with matrix calculations which are easier to compute [27].

Faddy and McClean (1999) used phase-type models to model LoS data for geriatric patients [12]. Fackrell (2009) gives an in-depth discussion on phase-type models and how they have been applied to healthcare processes [11]. The dis-advantage of phase-type models is that their generality can make the parameter estimation difficult and so a subclass of phase-type distributions called Coxian phase-type distribution were introduced [27].

\subsection{Coxian phase-type models}

Coxian phase-type models are a special type of phase-type distributions where the transient states in the system are ordered [27]. Therefore, to progress through the model the individual, here a patient, can either move to the next transient state sequentially or exit the system by passing into the absorbing state. Figure 2 illustrates a Coxian phase-type distribution and how a patient, can pass through the system.
The time spent by an individual, in each of the transient states can be found using the probability density function:

$f(t)=\mathbf{p} \exp \{\mathbf{Q} t\} \mathbf{q}$

where $\mathbf{p}=(1,0, \ldots, 0)$ is the probability of entering each of the transient states, $\mathbf{q}=\left(\mu_{1}, \mu_{2}, \ldots, \mu_{k}\right)^{\prime}$ is the probability of leaving the system, by being absorbed into the absorbing state, from each of the transient states and

$$
\mathbf{Q}=\left(\begin{array}{ccccc}
-\left(\lambda_{1}+\mu_{1}\right) & \lambda_{1} & 0 & \ldots & 0 \\
0 & -\left(\lambda_{2}+\mu_{2}\right) & \lambda_{2} & \ddots & \vdots \\
0 & 0 & \ddots & \ddots & 0 \\
\vdots & \ddots & \ddots & -\left(\lambda_{k-1}+\mu_{k-1}\right) & \lambda_{k-1} \\
0 & 0 & \ldots & 0 & -\mu_{k}
\end{array}\right)
$$

is the infinitesimal generator which provides all the transition rates between states. We note that $\mathbf{Q}$ here is a sub-matrix of the generator defined in Section 2.1 which excludes the first row and column. The $\lambda_{i}$ 's describe the transitions through the transient states, $S_{1}, \ldots, S_{k}$, and the $\mu_{i}$ 's represent the transitions into the absorbing state $S_{k+1}$.

Coxian phase-type distributions are commonly used to model healthcare pathways as transition through the transient states represents the progression of the patient through care and movement to the absorbing state represents a discharge process, such as the patient being discharged home from hospital or death. The two-term mixed exponential distribution described in Section 2.1 can be seen as a two-phase phase-type distribution. There are a number of advantages of using Coxian phase-type models to represent LoS, for example, they are mathematically tractable and do not over-parameterise the models which general phase-type distributions have a tendency to do [25].

Marshall and McClean (2004) used a Coxian phase-type distribution to represent LoS and analysed whether certain elderly patient character-istics contributed to them staying for a certain length of time in hospital [27]. Marshall and Zenga (2009) compared different approaches used to find the parameter estimates of phase-type distributions [31]. They found that the Quasi-Newton maximum likelihood approach was better in terms of convergence while the Nelder Mead algorithm performed better in terms of parameter estimation. They also discussed the recent developments of Coxian phase-type distributions within healthcare.
Fig. 2 A Coxian phase-type distribution

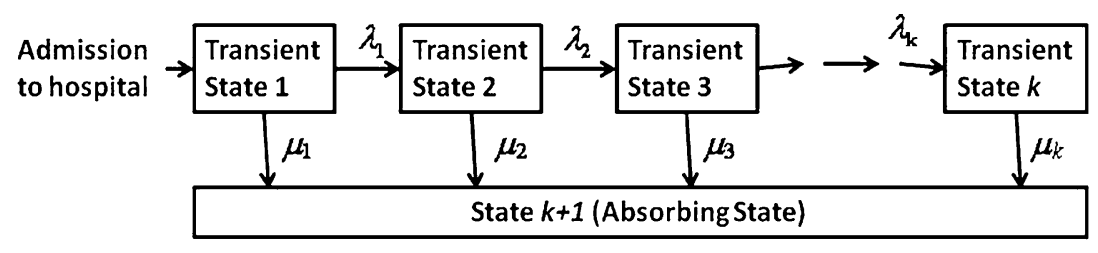




\subsection{Conditional phase-type model}

Conditional phase-type models extend the original phasetype model to include extra information about the random variable; from a healthcare point of view this could be a patient, $[3,27,28]$. This is accomplished by introducing a conditional com-ponent to the model, which groups the patients together using techniques so that patients with similar characteristics are in the same group. The phasetype distribution is then used to calculate the length of time the patient spends in each phase of the model. The techniques used to achieve this include, Classification and Regression trees [5], Bayesian Belief Networks [16, 27] and phase-type survival trees $[14,15]$. Figure 3 shows that the conditional component is implemented before the Coxian phase-type distribution and includes some techniques which can be used for the conditional component.

Marshall, et al. $(2003,2004)$ used a Bayesian belief network, which is a probabilistic graphical model, as the conditional component of a conditional phase-type model when analysing the LoS of geriatric patients in hospital [27, 28]. McClean et al. (2005) also used a conditional phasetype model when analysing geriatric patients. However, the conditional component in this case was a clustering technique where the patients were clustered based on the number of phases or states the patient passed through [26].

In this paper we require to determine if the benefits of thrombolysis balance the costs of treating patients and to see if the number of eligible patients who receive thrombolysis could be increased. To achieve this an analytic model is created which extends the approach of using a mixture of Coxian phase-type models with multiple absorbing states, as developed by McClean, et al. (2011) [25], to create a novel costs model for stroke patients. This methodology uses multiple Coxian phase-type models in parallel where each of these (parallel) sub-models is a separate pathway determined by patient characteristics including admission profiles and similar LoS profile. Within this framework, we can therefore incorporate the different parallel sub-models into a general phase-type model, where each sub-model constitutes a separate class (using the terminology of Markov models) and transitions between Coxian sub-phase-type models of different classes are never possible [25]. Our methodology then involves adding three costs together; the new drug cost, the hospital cost and the community care cost. Using an analytic approach allows for easy implementation and a fast evaluation of basic changes that hospital managers could make.

\section{The analytical model}

Our approach is an extension of the methodology described by McClean, et al. (2011) [25], to include costs. Multiple phase-type models are used to represent patients who follow several different pathways through a healthcare facility. This is achieved by clustering patients on arrival into homogeneous groups based on their LoS in hospital. For this paper we have used survival analysis to cluster patients, as in [25], but other approaches such as Bayesian belief networks $[27$, 28], conditional phase-type distributions or phase-type survival trees can be utilised instead. By constructing a phase-type model in this way we can overcome the identifiability and estimation problems of phase-type models while retaining their expressiveness and tractability.

In addition, the methodology includes several absorbing states which allow for the inclusion of different community care destinations such as rehabilitation and private nursing homes. In the following subsection we define the mixture of Coxian phase-type models with multiple absorbing states described by McClean et al. (2011) [25], before extending the approach to include costs in Section 3.2.

\subsection{A mixture of phase-type models with multiple} absorbing states

$C$ is defined as the number of transient classes and within each class $c$ there are $k_{c}$ phases, for $c=1, \ldots, C$.

Altogether there are $k=\sum_{c=1}^{c} k_{c}$ phases, where $S_{i}^{c}$ is the $i$ th phase of class $c: c=1, \ldots, C$ and $i=1, \ldots, k_{c}$. For this model we have $m$ absorbing states, $S_{k+1}, \ldots, S_{k+m}$. Transitions that occur between the state $S_{i}^{c}(i=1,2, \ldots, k-1)$ and $S_{i+1}{ }^{\mathrm{c}}$ have
Fig. 3 The Conditional Coxian phase-type distribution

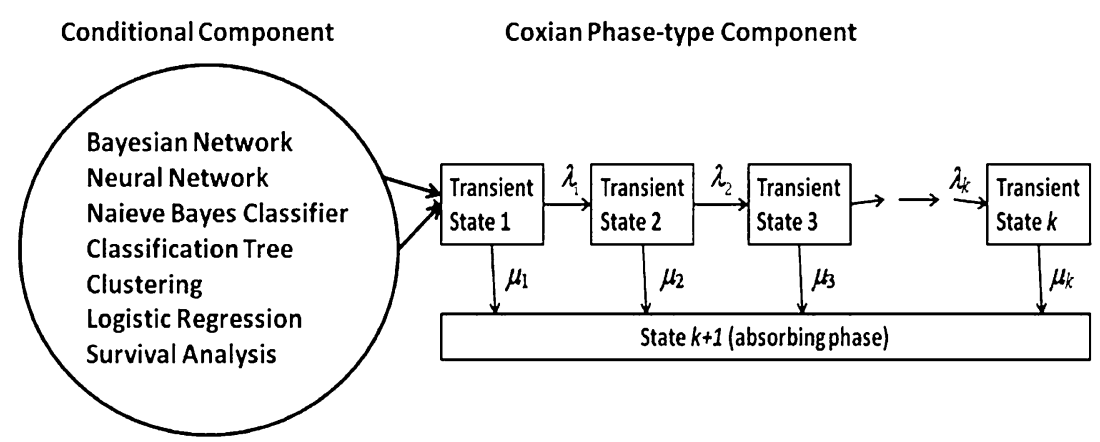


the transition rate $\lambda_{i}^{c}$ and other transitions between states of different transient classes are never possible. Transition can also take place from $S_{i}^{c}$ to any absorbing state $S_{m+j}$ with transition rate $\mu_{i j}{ }^{c}$ for $i=1, \ldots, k_{c}, j=1, \ldots, m, c=1, \ldots, C$. An example of a multiple phase-type model with multiple absorbing states can be found in Fig. 4. For more information on this model see McClean, et al. (2011), [25].

\subsection{The analytic cost model}

The total expected cost per patient in our approach is calculated by adding together three separate costs; firstly the new drug cost, which in this paper will be the cost of administering thrombolysis, secondly the cost incurred in hospital and thirdly the community care cost. The calculations for these three costs will be discussed in the following subsections with the total cost equation found in Section 3.2.4 followed by two examples in Section 3.2.5. In addition, the methodology for finding the maximum cost of the drug before we can no longer justify administering thrombolysis is presented in Section 3.2.6.

In this paper the analytic model will be applied to stroke data and used to assess the impact and costs of thrombolysis on stroke patients. However the approach could be used to analyse the impact and costs of any new drug introduced within the healthcare service.

\subsubsection{New drug cost}

Let $\tau$ be the additional fixed cost of an eligible patient receiving a new drug compared to the standard treatment for that medical complaint in the healthcare facility. Let $\alpha$ be the probability of an eligible patient receiving the drug. This will be dependent on the resources that are available to the department. The expected cost per patient of receiving the drug is therefore given by:

$G=\alpha \tau$

\subsubsection{Hospital cost}

Let $b_{i}^{c} d t\left(i=1,2, \ldots, k_{c}\right)$ be the expected cost of treatment per patient in time $(t, t+d t]$ in the transient state $S_{i}^{c}$ of the hospital, for example this could be the expected cost in acute or long term care in hospital. For a mixture of Coxian phase-type models the cost matrix is therefore defined as:

$\mathbf{B}=\left(\begin{array}{cccc}\mathbf{b}_{1} & 0 & \ldots & 0 \\ 0 & \mathbf{b}_{2} & \ddots & \vdots \\ \vdots & \ddots & \ddots & 0 \\ 0 & \ldots & 0 & \mathbf{b}_{C}\end{array}\right)$,

where $\mathbf{b}_{\mathrm{c}}=\left(b_{1}^{c}, b_{2}^{c}, \ldots, b_{k c}^{c}\right)^{\prime}$.

The vector of expected cost per patient in each state in hospital in the interval $(t, t+d t]$ is then given by:

$\mathbf{h}(t)=\mathbf{p} \exp (\mathbf{Q} t) \mathbf{B} d t$

where $\mathbf{p}=\left(\mathbf{p}_{1}, \mathbf{p}_{2}, \ldots, \mathbf{p}_{C}\right)$ is the entry row vector, partitioned into $C$ sub-vectors. The $c$ th sub-vector, $\mathbf{p}_{\mathrm{c}}$ has first element $\pi_{c}$ which is the probability of entering phase 1 of the corresponding class and the other elements are zero. Therefore, $\mathbf{p}_{c}=\left(\pi_{c}, 0, \ldots, 0\right)$.

$\mathbf{Q}$ is the transition matrix where,

$$
\mathbf{Q}=\left(\begin{array}{cccc}
\mathbf{Q}_{1} & 0 & \cdots & 0 \\
0 & \mathbf{Q}_{2} & \ddots & \vdots \\
\vdots & \ddots & \ddots & 0 \\
0 & \cdots & 0 & \mathbf{Q}_{c}
\end{array}\right)
$$

Fig. 4 An example of pathways/classes through a healthcare facility

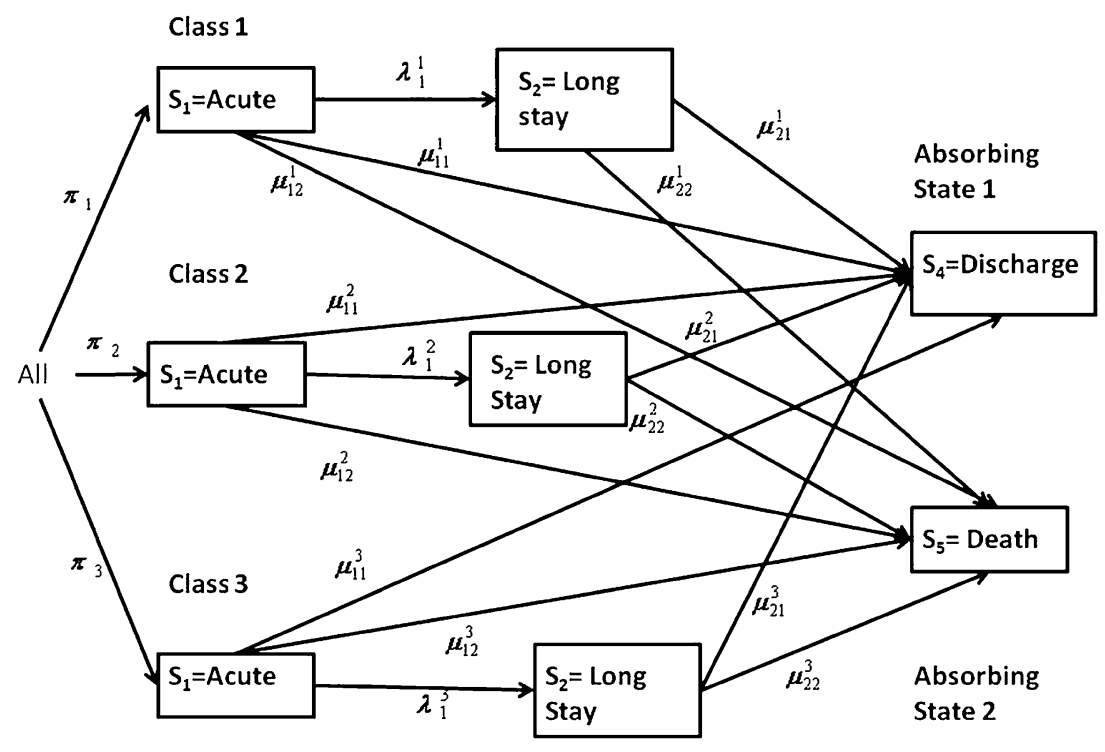


which consists of the sub-transition matrices corresponding to each class.

$\mathbf{Q}_{c}$ is then the transition matrix corresponding to class $C$ and is defined as:

$\mathbf{Q}_{c}=\left(\begin{array}{ccccc}-\left(\lambda_{1}^{c}+\sum \mu_{1 j}^{c}\right) & \lambda_{1}^{c} & 0 & \cdots & 0 \\ 0 & -\left(\lambda_{2}^{c}+\sum \mu_{2 j}^{c}\right) & \lambda_{2}^{c} & \ddots & \vdots \\ 0 & \ddots & \ddots & \ddots & 0 \\ \vdots & \ddots & \ddots & \ddots & \lambda_{k_{c}-1}^{c} \\ 0 & \cdots & 0 & 0 & -\sum \mu_{k_{c} j}^{c}\end{array}\right)$.

To find the total cost for each state, given by the column vector $\mathbf{h}(\mathrm{t})$, in the hospital we integrate over the interval $[0, \infty]$ to give $\mathbf{h}$.

$h=\int_{0}^{\infty} p \exp (Q t) B d t=[p \exp (Q t) B]_{0}^{\infty}=-p Q^{-1} B$

To find the total expected cost in hospital, $H$, we then multiply the vector of costs $\mathbf{h}$ by $\mathbf{e}$ which is a vector of $k 1$ 's. Therefore the total cost of hospital care is calculated using:

$H=-\mathbf{p} \mathbf{Q}^{-1} \mathbf{B e}$.

\subsubsection{The cost of community care}

Let $\mathbf{a}$ be the limiting vector of probabilities of eventual absorption into the absorbing phase $S_{k+1}$ for $i=1, \ldots, m$. This is found using the equation:

$\mathbf{a}=-\sum_{c=1}^{C} \mathbf{p}_{C} \mathbf{Q}_{C}^{-1} \mathbf{q}^{C}$

given in [20]. The vector $\mathbf{p}_{C}$ is the entry row vector to class $C$ and matrix $\mathbf{Q}_{C}$ is the transition matrix corresponding to class $C$ (see Section 2.3) and $\mathbf{q}=\left(\mathbf{q}^{1}, \mathbf{q}^{2}, \ldots, \mathbf{q}^{\mathrm{C}}\right)^{\prime}$ where,

$\mathbf{q}^{C}=\left(\begin{array}{ccc}\mu_{11}^{C} & \cdots & \mu_{1 m}^{C} \\ \vdots & & \\ \mu_{k_{c} 1}^{C} & & \mu_{k_{c} m}^{C}\end{array}\right)$

is the matrix of absorbing probabilities for the corresponding classes.
The cost of treatment in each of the absorbing phases is $d_{k+i}$. Therefore the cost of community care is given by:

$\mathbf{w}=\mathbf{a D}$

Where $\mathbf{D}$ is the cost matrix:

$\mathbf{D}=\left(\begin{array}{cccc}d_{m+1} & 0 & \cdots & 0 \\ 0 & d_{m+2} & \ddots & \vdots \\ \vdots & \ddots & \ddots & 0 \\ 0 & \cdots & 0 & d_{m+i}\end{array}\right)$.

To find the total cost of community care the vector $\mathbf{w}$ is multiplied by e which is a vector of $m 1 \dot{s}$. Therefore, the total cost of community care is:

$W=\mathbf{a D e}$

\subsubsection{Total cost}

Putting Eqs. 1, 3 and 5 together we find that the total expected cost per person for patients who are eligible to receive a new treatment is given by:

$\bar{c}=\alpha \tau-\mathbf{p} \mathbf{Q}^{-1} \mathbf{B e}+\mathbf{a D e}$.

i.e.

$\bar{c}=G+H+W$

\subsubsection{Illustrative examples}

In the following section the approach has been applied to a simple two phase phase-type model with one absorbing state in Example 2. In addition it has also been applied to a model which has two classes each consisting of two transient states and two absorbing states in Example 3.

Example 2: We consider the cost of a patient who is eligible to receive a new treatment moving through a system with two transient states $S_{1}$ (Acute care) and $\mathrm{S}_{2}$ (Long term care) and one absorbing state $\mathrm{S}_{3}$ (Discharge/Death). See (Fig. 5.).
Fig. 5 A system with one class containing two transient states and one absorbing state

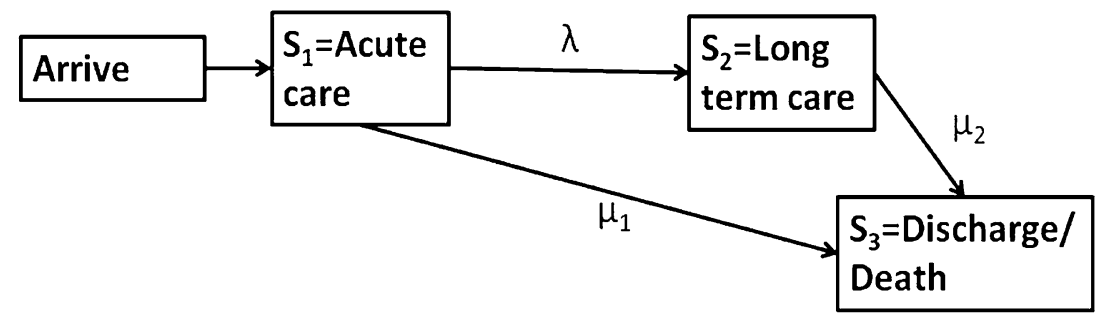


Using Eq. 1 the expected cost of this patient receiving the initial treatment is:

$G=\alpha^{*} \tau$

In this case the initial probability is $\mathbf{p}=$ $(1,0)$. The transition matrix is

$$
\begin{array}{r}
\mathbf{Q}=\left[\begin{array}{cc}
-\left(\lambda+\mu_{1}\right) & \lambda \\
0 & -\mu_{2}
\end{array}\right], \\
\mathbf{Q}^{-1}=\left[\begin{array}{cc}
-1 /\left(\lambda+\mu_{1}\right) & -\lambda /\left(\lambda+\mu_{1}\right) \mu_{2} \\
0 & -1 / \mu_{2}
\end{array}\right]
\end{array}
$$

and $\mathbf{B}=\left(b_{1}, b_{2}\right)^{\prime}$.

So using Eq. 2 the cost of treatment in the hospital is:

$$
\begin{aligned}
\mathbf{h} & =-\left(\begin{array}{ll}
\frac{-1}{\left(\lambda+\mu_{1}\right)} & \frac{-\lambda_{1}}{\left(\lambda+\mu_{1}\right) \mu_{2}}
\end{array}\right)\left(\begin{array}{c}
b_{1} \\
b_{2}
\end{array}\right) \\
& =\frac{b_{1}}{\lambda+\mu_{1}}+\frac{b_{2} \lambda}{\left(\lambda+\mu_{1}\right) \mu_{2}}=b_{1} \bar{T}_{1}+b_{2} h_{1} \bar{T}_{2},
\end{aligned}
$$

Where $\bar{T}_{1}$ is the mean duration in $S_{1}, \bar{T}_{2}$ is the mean duration in $S_{2}, h_{1}=\frac{\lambda}{\lambda+\mu_{1}}$ is the probability that the patient enters $S_{2}$ and $b_{i}$ is the unit cost per patient in $S_{i}$. In this case we only have one absorbing state so the expansion of Eq. 3 is a scalar.

The cost of going to the community care, which is the absorbing state, is $d$. Using Eq. 4 we find that the cost of community care is:

$\mathbf{w}=1 * d=d$

as the probability of the patient going to the absorbing state death/discharge is 1 and the cost of care in this absorbing state is $d$.

Combining these equations we find that the total expected cost of a patient is:

$\overline{\mathbf{c}}=\alpha \tau+\left(b_{1} \bar{T}_{1}+b_{2} h_{1} \bar{T}_{2}\right)+d$

Example 3: We consider the total cost of treating patients when there are two classes, $c_{1}$ (receiving the drug) and $c_{2}$ (receiving the standard treatment). The extra cost of receiving treatment in class 1 is $\tau$ and there is no extra cost of receiving treatment in class 2 . Within each class there are two transient states $\mathrm{S}_{1}$ (Acute Care) and $\mathrm{S}_{2}$ (Long term Care) in hospital and two absorbing states $\mathrm{S}_{3}$ (Discharged Home) and $\mathrm{S}_{4}$ (Death) for community care. (See Fig. 6).

The extra cost of the new treatment in this example is $G=\alpha \tau$.

The probability vector of going to the transient states in the hospital after receiving either the new treatment or standard treatment is $\mathbf{p}=((1,0),(1,0))$. The transition matrix is:

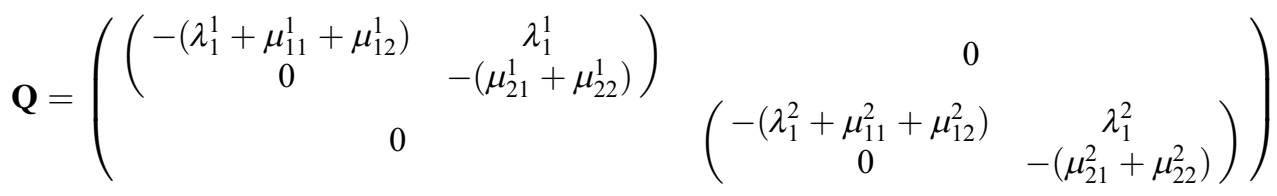

and the cost of being in each of the transient states in the hospital is:

$\mathbf{B}=\left(\begin{array}{cc}\left(\begin{array}{c}b_{1}^{1} \\ b_{2}^{1}\end{array}\right) & 0 \\ 0 & \left(\begin{array}{c}b_{1}^{2} \\ b_{2}^{2}\end{array}\right)\end{array}\right)$

Therefore, the total cost in hospital is:

$$
\begin{aligned}
H= & \frac{b_{1}^{1}}{\lambda_{1}^{1}+\mu_{11}^{1}+\mu_{12}^{1}}+\frac{b_{2}^{1} \lambda_{1}^{1}}{\left(\lambda_{1}^{1}+\mu_{11}^{1}+\mu_{12}^{1}\right)\left(\mu_{21}^{1}+\mu_{22}^{1}\right)} \\
& +\frac{b_{1}^{2}}{\lambda_{1}^{2}+\mu_{11}^{1}+\mu_{12}^{1}}+\frac{b_{2}^{2} \lambda_{1}^{2}}{\left(\lambda_{1}^{2}+\mu_{11}^{2}+\mu_{12}^{2}\right)\left(\mu_{21}^{2}+\mu_{22}^{2}\right)}
\end{aligned}
$$

The probability of going to each of the absorbing states (community care) is:

$\mathbf{q}=\left(\begin{array}{l}\left(\begin{array}{ll}\mu_{11}^{1} & \mu_{12}^{1} \\ \mu_{21}^{1} & \mu_{22}^{1}\end{array}\right) \\ \left(\begin{array}{ll}\mu_{11}^{2} & \mu_{12}^{2} \\ \mu_{21}^{2} & \mu_{22}^{2}\end{array}\right)\end{array}\right)$

The cost of treatment in each of the states:

$\mathbf{D}=\left(\begin{array}{rr}d_{1} & 0 \\ 0 & d_{2}\end{array}\right)$.

Using Eq. 4 the cost of community care is therefore, 
Fig. 6 Two classes each consisting of two transient states and two absorbing states

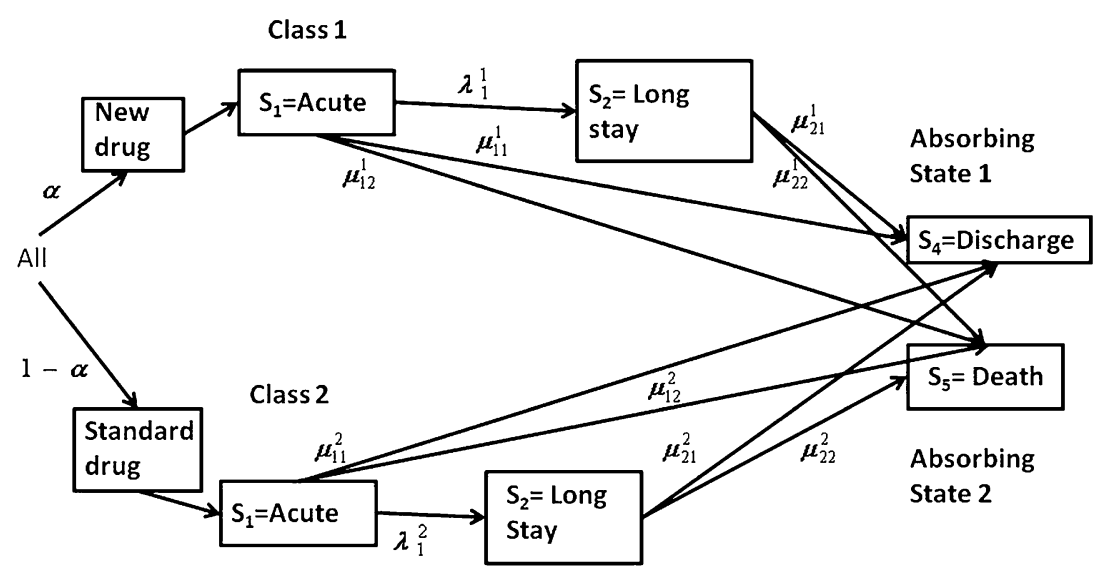

$W=d_{1}\left(\frac{\mu_{11}^{1}}{\lambda_{1}^{1}+\mu_{11}^{1}+\mu_{12}^{1}}+\frac{\lambda_{1}^{1} \mu_{21}^{1}}{\left(\lambda_{1}^{1}+\mu_{11}^{1}+\mu_{12}^{1}\right)\left(\mu_{21}^{1}+\mu_{22}^{1}\right)}\right.$

$\left.+\frac{\mu_{11}^{2}}{\lambda_{1}^{2}+\mu_{11}^{2}+\mu_{12}^{2}}+\frac{\lambda_{1}^{2} \mu_{21}^{2}}{\left(\lambda_{1}^{2}+\mu_{11}^{2}+\mu_{12}^{2}\right)\left(\mu_{21}^{2}+\mu_{22}^{2}\right)}\right)+$

$d_{2}\left(\frac{\mu_{11}^{1}}{\lambda_{1}^{1}+\mu_{11}^{1}+\mu_{12}^{1}}+\frac{\lambda_{1}^{1} \mu_{21}^{1}}{\left(\lambda_{1}^{1}+\mu_{11}^{1}+\mu_{12}^{1}\right)\left(\mu_{21}^{1}+\mu_{22}^{1}\right)}\right.$

$\left.+\frac{\mu_{11}^{2}}{\lambda_{1}^{2}+\mu_{11}^{2}+\mu_{12}^{2}}+\frac{\lambda_{1}^{2} \mu_{21}^{2}}{\left(\lambda_{1}^{2}+\mu_{11}^{2}+\mu_{12}^{2}\right)\left(\mu_{21}^{2}+\mu_{22}^{2}\right)}\right)$

By adding together $G, H$ and $W$ the total cost in Example 4 can be found.

$$
\begin{aligned}
& \bar{c}=\alpha \tau+\frac{b_{1}^{1}}{\lambda_{1}^{1}+\mu_{11}^{1}+\mu_{12}^{1}}+\frac{b_{2}^{1} \lambda_{1}^{1}}{\left(\lambda_{1}^{1}+\mu_{11}^{1}+\mu_{12}^{1}\right)\left(\mu_{21}^{1}+\mu_{22}^{1}\right)} \\
& +\frac{b_{1}^{2}}{\lambda_{1}^{2}+\mu_{11}^{1}+\mu_{12}^{1}}+\frac{b_{2}^{2} \lambda_{1}^{2}}{\left(\lambda_{1}^{2}+\mu_{11}^{2}+\mu_{12}^{2}\right)\left(\mu_{21}^{2}+\mu_{22}^{2}\right)}+ \\
& d_{1}\left(\frac{\mu_{11}^{1}}{\lambda_{1}^{1}+\mu_{11}^{1}+\mu_{12}^{1}}+\frac{\lambda_{1}^{1} \mu_{21}^{1}}{\left(\lambda_{1}^{1}+\mu_{11}^{1}+\mu_{12}^{1}\right)\left(\mu_{21}^{1}+\mu_{22}^{1}\right)}\right. \\
& \left.+\frac{\mu_{11}^{2}}{\lambda_{1}^{2}+\mu_{11}^{2}+\mu_{12}^{2}}+\frac{\lambda_{1}^{2} \mu_{21}^{2}}{\left(\lambda_{1}^{2}+\mu_{11}^{2}+\mu_{12}^{2}\right)\left(\mu_{21}^{2}+\mu_{22}^{2}\right)}\right)+ \\
& d_{2}\left(\frac{\mu_{11}^{1}}{\lambda_{1}^{1}+\mu_{11}^{1}+\mu_{12}^{1}}+\frac{\lambda_{1}^{1} \mu_{21}^{1}}{\left(\lambda_{1}^{1}+\mu_{11}^{1}+\mu_{12}^{1}\right)\left(\mu_{21}^{1}+\mu_{22}^{1}\right)}\right. \\
& \left.+\frac{\mu_{11}^{2}}{\lambda_{1}^{2}+\mu_{11}^{2}+\mu_{12}^{2}}+\frac{\lambda_{1}^{2} \mu_{21}^{2}}{\left(\lambda_{1}^{2}+\mu_{11}^{2}+\mu_{12}^{2}\right)\left(\mu_{21}^{2}+\mu_{22}^{2}\right)}\right)
\end{aligned}
$$

\subsubsection{Maximum cost of new drug}

Using the total cost per person we have also been able to calculate the maximum cost of thrombolytic therapy before the benefits no longer outweigh the costs of administration.

Let $\mathbf{p}_{T}$ and $\mathbf{p}_{N T}$ be the initial probability vectors to the system when a proportion of patients receive the thrombolytic therapy and when no patients receive the thrombolytic therapy respectively. Let $\mathbf{Q}_{T}^{-1}$ and $\mathbf{Q}_{N T}{ }^{-1}$ be the transition matrix when a proportion of patients receive the thrombolytic therapy and when no patients receive the thrombolytic therapy respectively. Also, let $\mathbf{a}_{T}$ and $\mathbf{a}_{N T}$ be the limiting vector of probabilities of eventual absorption when a proportion of patients receive the thrombolytic therapy and when no patients receive the thrombolytic therapy respectively.

The total cost when a proportion of patients receive thrombolytic therapy is $c_{T}$ where:

$\bar{c}_{T}=\alpha \tau-\mathbf{p}_{T} \mathbf{Q}_{T}{ }^{-1} \mathbf{B e}+\mathbf{a}_{T} \mathbf{D e}$

The total cost when no patients receive thrombolytic therapy is $c_{N T}$ where:

$c_{N T}=-\mathbf{p}_{N T} \mathbf{Q}_{N T}{ }^{-1} \mathbf{B e}+\mathbf{a}_{N T} \mathbf{D e}$

When the thrombolysis cost is the same as the cost when there is no thrombolysis administered $\bar{c}_{T}$ will equal $\bar{c}_{N T}$, therefore,

$$
\begin{aligned}
\alpha \tau-\mathbf{p}_{T} \mathbf{Q}_{T}{ }^{-1} \mathbf{B e}+\mathbf{a}_{T} \mathbf{D e}= & -\mathbf{p}_{N T} \mathbf{Q}_{N T}{ }^{-1} \mathbf{b e} \\
& +\mathbf{a}_{N T} \mathbf{D e}
\end{aligned}
$$

This simplifies to

$\alpha \tau=-\mathbf{p}_{N T} \mathbf{Q}_{N T}^{-1} \mathbf{B e}+\mathbf{a}_{N T} \mathbf{D e}+\mathbf{p}_{T} \mathbf{Q}_{T}^{-1} \mathbf{B e}-\mathbf{a}_{T} \mathbf{D e}$

Therefore,

$$
\tau=\left(\left(\mathbf{p}_{T} \mathbf{Q}_{T}^{-1}-\mathbf{p}_{N T} \mathbf{Q}_{N T}{ }^{-1}\right) \mathbf{B e}+\left(\mathbf{a}_{N T}-\mathbf{a}_{T}\right) \mathbf{D e}\right) / \alpha
$$

\section{The application}

This methodology was applied to 5 year retrospective data for patients admitted to the Belfast City Hospital with a diagnosis of stroke (cerebral haemorrhage-bleed in the brain; cerebral infarction - clot on the brain; transient ischemic attack - minor stroke; and unspecified or undetermined type of stroke). The data was taken from the Patient Administration System (PAS) - a computerized system that records patient activity relating to inpatients, outpatients, and waiting lists, A\&E and case note tracking. The data contained information including age, gender, diagnosis, LoS and destination on discharge. There were 154 (7.8\%) 
patients admitted with cerebral haemorrhage, $655(33 \%)$ with cerebral infarction, $425(21.4 \%)$ with transient ischemic attack and $751(37.8 \%)$ with an unspecified stroke type.

Thrombolysis is a therapy which uses a clot dispersing drug. It has the potential to reduce the short term institutionalisation of certain types of stroke patients. Although the method described above can be used for all stroke patients, in this paper we will concentrate on patients who are eligible to receive thrombolysis (see Section 1). The number of patients who receive the new drug, thrombolysis, is dependent on the resources that are available. This allows us to determine if the benefits of thrombolytic therapy balance out the cost of administering the expensive drug. Once the dataset was analysed and the patients who were eligible to receive the drug were identified, two new groups were created, 'Receive Thrombolysis' and 'Don't receive Thrombolysis'.

The methodology described in Section 3 is a mixture of Coxian phase-type models with multiple absorbing states as there are many treatment pathways a patient can take through a hospital or department. We here use survival analysis to cluster the LoS data of patients into homogeneous groups on arrival. Survival analysis is defined as the measure of time from a starting point to when an event occurs, for example this could be the time from when a patient is admitted to hospital to the time the patient is discharged. LoS data are ideally suited to survival analysis as they tend not to have a Gaussian distribution and are often censored, which means that other statistical approaches, typically based on Gaussian assumptions cannot be applied to them [14]. Survival analysis was performed on the patients who were eligible to receive the thrombolytic therapy using LoS and destination as outcome measures. Survival probabilities were found using KaplanMeier estimation and log ranks tests to determine if the LoS in hospital affected the patients discharge destination. The Kaplan-Meier estimates can be seen in Fig. 7 [25].

The log rank test showed that the relationship between LoS of patients who were eligible to receive thrombolysis and their discharge destination was significant $\left(\chi^{2}\right.$ statistic 29.22, $p=0.000$ ), therefore, six new absorbing states were created to incorporate the possible destinations (usual residence, private nursing home and death for those who receive thrombolysis and usual residence, private nursing home and death for those who do not receive thrombolysis).

Usual residence for those who received and did not receive thrombolysis were further split into those who went to their usual residence and those who went to their usual residence but also require rehabilitation from the SouthEast Belfast stroke scheme. A diagram showing how the model is structured can be found in Fig. 8 .

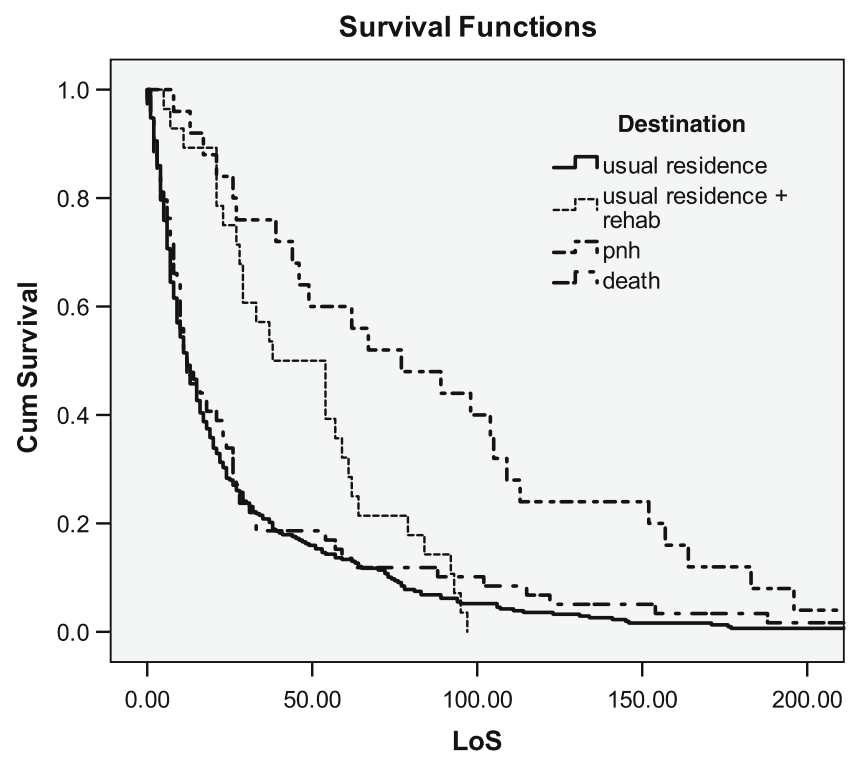

Fig. 7 The Kaplan-Meier estimates for destination distributions

After the pathways have been established, a penalized likelihood approach was used to find the optimal number of phases required to represent the distribution of patients in hospital for each pathway. Such an approach includes a penalty which prohibits the parameters from being over-fitted [17].

Maximum likelihood for a phase-type model with a given number of phases was calculated using the freely downloadable package EMpht [2, 35] with the corresponding length of stay data. EMpht uses the expectation-maximisation (EM) algorithm [7] and is a very efficient tool for parameter estimation of phase-type distributions [11]. Our penalised likelihood approach progressively increases the number of phases $(\mathrm{K})$ until the Akaike information criterion (AIC) achieves a minimum. For each value of $\mathrm{K}$ :

$\mathrm{AIC}=2 \mathrm{C}-2(\mathrm{~L}(\Theta) \mid \mathrm{X})$,

where $\mathrm{C}$ are the degrees of freedom (number of free parameters of the distribution) and $\mathrm{L}(\Theta \mid \mathrm{X})$ is the maximised $\log$ likelihood function for $\mathrm{C}$ parameters [1]. As each phase, except the last one, requires only 2 parameters for estimation and the last phase requires one parameter estimation, the degrees of freedom in our model are $\mathrm{C}=$ $2 \mathrm{~K}-1$ [13, 16, 25] when there are $\mathrm{K}$ phases.

For eligible stroke patients it was found that two phases in the Coxian phase-type distribution were sufficient to model LoS in each class. This may be due to clustering the data thus mitigating against heterogeneity. In this model we end up with eight absorbing states namely usual residence for those who receive and do not receive thrombolysis each of whom have two options; to go to their usual residence without any extra treatment or go to their usual residence with a rehabilitation program. Therefore the 8 absorbing 
Fig. 8 A diagram of how the model is structured

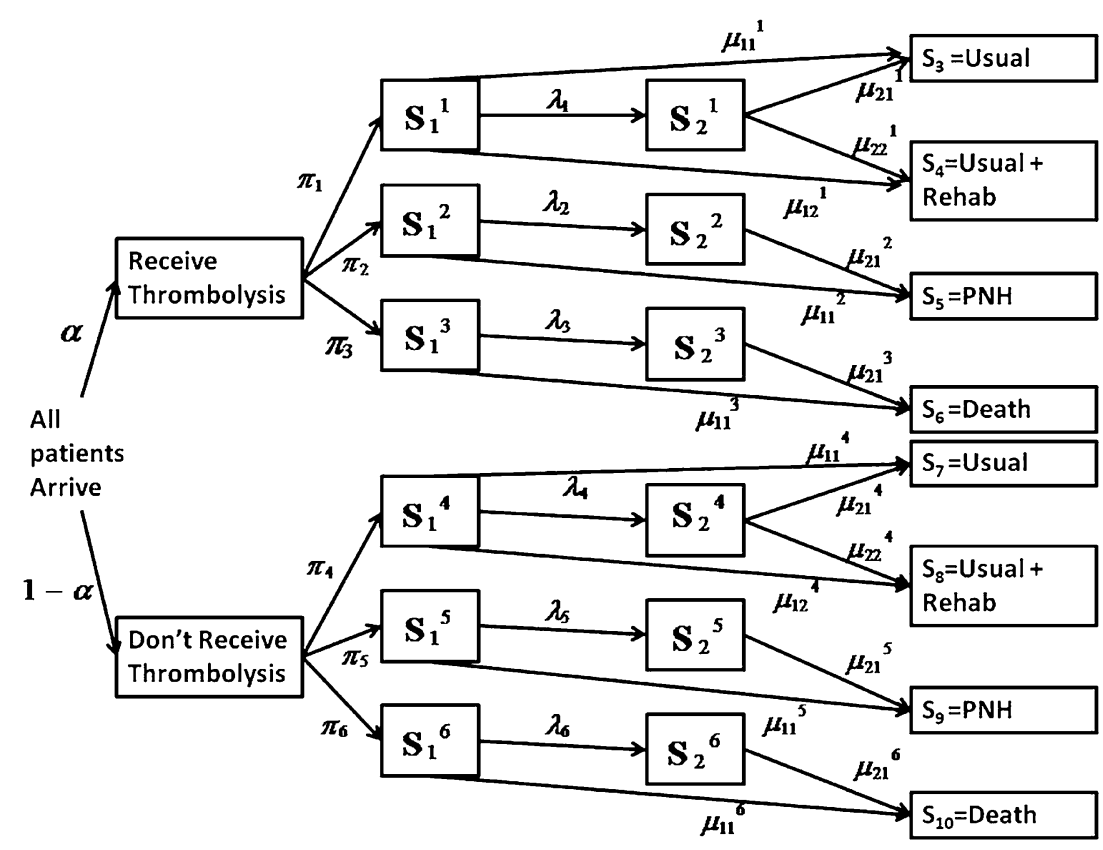

states are: usual residence, usual residence with rehabilitation programme, private nursing home and death for those who receive thrombolysis and usual residence, usual residence with rehabilitation program, private nursing home and death for those who do not receive thrombolysis.

Although an exact value for the extra cost of thrombolytic treatment per patient is hard to find, Sandercock (2006), [38], suggests that a reasonable approximation is $£ 750$ per person which includes the cost of the drug at $£ 450$ and extra time required by more experienced staff. The mean LoS in hospital is based on the Belfast City Hospital records and for those patients who receive thrombolysis the LoS in acute care is estimated to be reduced by 2 days or $15 \%$ [39]. The cost of acute care is approximated as $£ 164.80$ per person per day and on-going care costing $£ 114.80$ per person per day [36]. For patients admitted to usual residence with rehabilitation the estimated amount of time needed in rehabilitative care is $26 \mathrm{~h}$ (data from stroke patients admitted to the SouthEast Belfast stroke scheme) and a cost of $£ 38$ per hour of client contact is assigned. The mean length of time that a patient stays in private nursing home is estimated to be 11.9 months [3] so the cost is $£ 2490.76$ per month. We estimate the time spent in private nursing home using mortality rates. This is because most patients leave institutional care by death and although a few are admitted back to hospital they are usually terminal patients [41].

\section{The results}

The results (Fig. 9) show that as the proportion of eligible patients who receive thrombolysis increases the total cost per patient decreases. When no patients receive thrombolysis the total cost per person is $£ 6363.2$ and when $50 \%$ of eligible patients receive thrombolysis the total cost per person is $£ 6252.4$ which is a saving of $£ 110.8$ per person. This indicates that the benefits of thrombolysis, which reduces the LoS in hospital and the proportion of people who go to private nursing homes, outweigh the expense of administering the drug.

This model shows that by increasing the proportion of people who receive thrombolysis the department can become more efficient and cost-effective. Using Eq. 11 the maximum cost of thrombolytic therapy before the benefits no longer outweigh the costs of administering the drug is $£ 971.59$. In other words, using the expected cost we

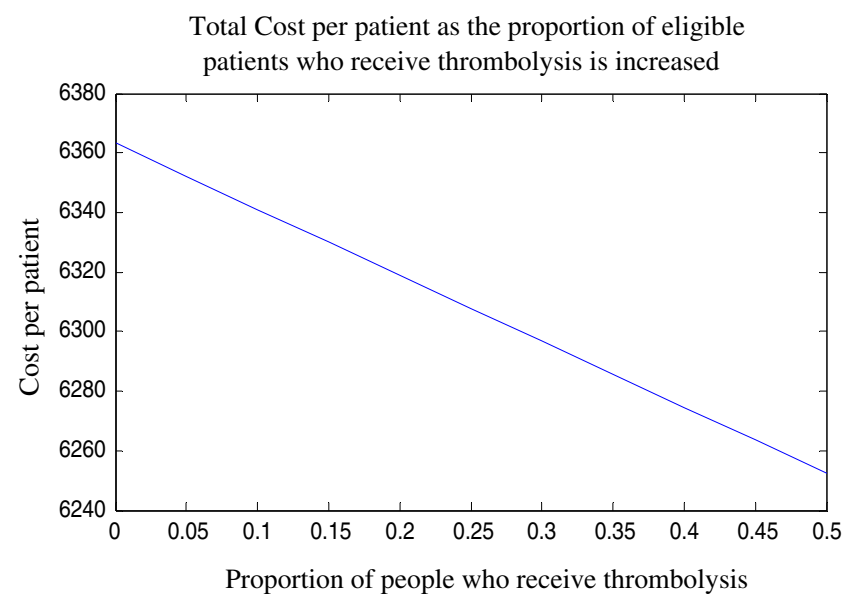

Fig. 9 The cost per person as the proportion of eligible patients who receive thrombolytic therapy increases 
would still administer treatment as long as the cost of the treatment remained under $£ 971$.

\section{Conclusions}

The approach presented in McClean et al. (2011) [25], can be used to incorporate extra absorbing states and pathways through the hospital to give a realistic representation of patient flow through a healthcare environment. With a growing emphasis on the efficiency and cost-effectiveness of healthcare, the current model extends our previous approach [25] and looks more closely at patients who are eligible to receive a clot dispersing therapy called thrombolysis, which decreases the short term hospitalisation of eligible stroke patients. By introducing costs to the model we have shown that the extra expense of administering thrombolysis is balanced out by the decreased costs in hospital and community care. The model uses survival analysis to cluster the patients into groups before they move through a phase-type distribution and into absorbing phases which could be usual residence, usual residence with rehabilitation, private nursing home and death. Costs are attached to each part of the process so that the total cost per patient is found. By increasing the proportion of eligible patients who receive thrombolysis we can see a gradual decrease in the cost per person. However, if the cost of thrombolytic therapy is increased to a value greater than $£ 971.59$ then the benefits of the drug will no longer balance the cost of administering the drug and new analysis will need to be carried out to assess the cost effectiveness in terms of cost per improvement in quality of life.

Acknowledgements The authors acknowledge support for this work from the Engineering and Physical Sciences Research Council (Grant References EP/E019900/1 and GR/S29874/01).

Any views or opinions presented herein are those of the authors and do not necessarily represent those of RIGHT or MATCH, their associates or their sponsors. Support has also been received from the Northern Ireland Health and Social Care Research \& Development (HSC R\&D) Office.

\section{References}

1. Akaike H (1974) A new look at the statistical model identification. IEEE T Automat Contr 19(6):716-723

2. Assmussen S, Nerman O, Olsson M (1006) Fitting phase-type distributions via the EM algorithm. Scand J Stat 23:419-441

3. Bebbington A, Darton R, Netton A (2001) Care homes for older people: volume 2 admissions, needs and outcomes, The 1995-96 National Longitudinal Survey of Publicly-funded Admissions Edn, Personal Social Services Research Unit, University of Kent at Canterbury

4. Caulkins JP, Dietze P, Ritter A (2007) Dynamic Compartmental model of trends in Australian drug use. Health Care Manag Sci 10:151-162
5. Christodoulou G, Taylor GJ (2001) Using a continuous time hidden Markov process, with covariates, to model bed occupancy of people aged over 65 years. Health Care Manag Sci 4:21-24

6. Cochran JK, Bharti A (2006) Stochastic bed balancing of an obstetrics hospital. Health Care Manag Sci 9:31-45

7. Dempster AP, Laird NM, Rubin DB (1977) Maximum likelihood from incomplete data via the EM algorithm. J R Stat Soc Ser B 39 (1):1-38

8. Department of Health (2009) NHS Choices: Your Health, Your Choice: Overview Department of Health, http://www.nhs.uk/ NHSEngland/thenhs/about/Pages/overview.aspx. Accessed 24/01/ 11

9. Department of Health (2008) NHS Institute for Innovation and Improvement. Department of Health, http://www.institute.nhs.uk/. Accessed 25/01/11

10. Department of Health (2008) NHS Institute for Innovation and Improvement: Performance Management. Department of Health, http://www.institute.nhs.uk/quality_and_service_improvement_tools/quality_and_service_improvement_tools/performance_management.html. Accessed 25/01/11

11. Fackrell M (2009) Modelling healthcare systems with phase-type distributions. Health Care Manag Sci 12:11-26

12. Faddy MJ, McClean SI (1999) Analysing data on lengths of stay of hospital patients using phase-type distributions. Appl Stoch Model Bus Ind 15:311-317

13. Faddy MJ, McClean SI (2005) Markov Chain Modelling for geriatric patient care. Methods Inf Med 44:369-373

14. Garg L, McClean SI, Meenan BJ, El-Darzi E, Millard PH (2009) Clustering patient length of stay using mixtures of Gaussian models and phase type distributions. 22nd IEEE Symposium on Computer-Based Medical Systems (CBMS 2009), Albuquerque, New Mexico, USA, August 3-4, 2009, pp 1-7

15. Garg L, McClean S, Meenan, B and Millard P (2009) A phase type survival tree model for clustering patient Length of Stay. Applied Stochastic Models and Data Analysis. The XIII International Conference, Vilnius, Lithuania, pp 477

16. Garg L, McClean S, Meenan B, Millard P (2010) A nonhomogeneous discrete time Markov model for admission scheduling and resource planning in a cost or capacity constrained healthcare system. Health Care Manag Sci 13:155-169

17. Green PJ (1987) Penalized likelihood for general semi-parametric regression models. Int Stat Rev 55:245-259

18. Harper PR (2002) A Framework for Operational Modelling of Hospital Resources. Health Care Manag Sci 5:165-173

19. Harrison GW (2001) Implications of mixed exponential occupancy distributions and patient flow models for health care planning. Health Care Manag Sci 4:35-45

20. Jones PW, Smith P (2001) Stochastic processes: an introduction. Arnold, London

21. Latouche G, Ramaswami V (1999) Introduction to matrix analytic methods in stochastic modelling. SIAM, Philadelphia

22. McClean SI, McAlea B, Millard PH (1998) Using a Markov reward model to estimate spend down costs for a geriatric department. J Oper Res 49:1021-1025

23. McClean SI, Millard P (2007) Where to treat the older patient? Can Markov models help us better understand the relationship between hospital and community care? J Oper Res Soc 58:255-261

24. McClean SI, Millard PH (1998) A Three compartment model of the patient flows in a geriatric department: a decision support approach. Health Care Manag Sci 1:159-163

25. McClean S, Barton M, Garg L, Fullerton K (2011) Combining markov models and discrete events simulation to plan stroke patient care. Transactions on Modeling and Computer Science (TOMACS) 21(4)

26. McClean S, Faddy M, Millard P (2005) Markov model-based clustering for efficient patient care. Proceedings of the 18th IEEE 
symposium on computer-based medical systems IEEE, Piscataway, pp 467

27. Marshall AH, McClean SI (2004) Using Coxian Phase-type distributions to identify patient characteristics for duration of stay in hospital. Health Care Manag Sci 7:285-289

28. Marshall AH, McClean SI (2003) Conditional phase-type distributions for modelling patient length of stay in hospital. Int Trans Oper Res 10:565-576

29. Marshall AH, McClean SI, Millard PH (2004) Addressing bed costs for the elderly: a new methodology for modelling patient outcomes and length of stay. Health Care Manag Sci 7:27-33

30. Marshall A, Vasilakis C, El-Darzi E (2005) Length of stay-based patient flow models: recent developments and future directions. Health Care Manag Sci 8:213-220

31. Marshall AH, Zenga M (2009) Recent developments in fitting coxian phase-type distributions in healthcare. Applied stochastic models and data analysis. The XIII International Conference, Vilnius, Lithuania, pp 482

32. Millard PH (1989) Geriatric medicine: a new method of measuring bed usage and a theory for planning, MD thesis, University of London

33. Neuts MF (1981) Matrix-geometric solutions in stochastic models: an algorithmic approach. John Hopkins University Press, Baltimore

34. Norris JR (1998) Markov chains. Cambridge Series in Statistical and Probabilistic Mathematics. Cambridge University Press, Cambridge, England
35. Olsson M (1996) Estimation of phase-type distributions from censored data. Scand J Stat 23:443-460

36. Royal College of Physicians National Sentinel Stroke Audit Report (2008) Audit of stroke, prepared for on behalf of the Intercollegiate Stroke Working Party, London, RCP

37. Saka O, McGuire A, Wolfe C (2009) Cost of stroke in the United Kingdom. Age Ageing 38:27-32

38. Sandercock P, Berge E, Dennis M, Forbes J, Hand P, Kwan J, Lewis S, Lindley R, Neilson A, Wardlaw J (2004) Costeffectiveness of Thrombolysis with Recombinant Tissue Plasminogen Activator for Acute Ischemic Stroke Assessed by a Model Based on UK NHS Costs 1490-1497

39. Sundburg G, Bagust A, Terent A (2003) A model for costs of stroke service. Health Policy 63:81-94

40. Taylor G, McClean S, Millard P (1998) Continuous time Markov model for geriatric patient behaviour. Appl Stoch Model Data Anal 13:315-323

41. The National Institute of Neurological Disorders and Stroke rt-PA Stroke Study Group (NINDS) (1995) New Engl J Med 33:15811588

42. The NHS Confederation (2010) On the Day Briefing- 2010 Budget Report, The NHS Conderation,http://www.nhsconfed.org/ Documents/budget_2010_briefingmarch10.pdf

43. Xie H, Chaussalet TJ, Millard PH (2006) A Model based approach to the analysis of patterns of length of stay in institutional long-term care. IEEE Trans Inf Tech Biosci 10:512551 